\title{
Article \\ GAN-LSTM Joint Network Applied to Seismic Array Noise Signal Recognition
}

\author{
Jian Li ${ }^{1, *} \mathbb{0}$, Dongwei Hei ${ }^{2}$, Gaofeng Cui ${ }^{3}$, Mengmin $\mathrm{He}^{3}$, Juan Wang ${ }^{1}$, Zhehan Liu ${ }^{1}$, Jie Shang ${ }^{1}$, \\ Xiaoming Wang ${ }^{1}$ and Weidong Wang ${ }^{3}$ \\ 1 CTBT Beijing National Data Center, Beijing 100085, China; wang.juan@ndc.org.cn (J.W.); \\ liu.zhehan@ndc.org.cn (Z.L.); shang.jie@ndc.org.cn (J.S.); wang.xiaoming@ndc.org.cn (X.W.) \\ 2 Northwest Institute of Nuclear Technology, Xi'an 710024, China; heidongwei@nint.ac.cn \\ 3 School of Electronic Engineering, Beijing University of Posts and Telecommunications, Beijing 100876, China; \\ cuigaofeng@bupt.edu.cn (G.C.); mengmin_he@bupt.edu.cn (M.H.); wangweidong@bupt.edu.cn (W.W.) \\ * Correspondence: lijian1980@bupt.edu.cn; Tel.: +86-10-6119-8389
}

check for updates

Citation: Li, J.; Hei, D.; Cui, G.; He, M.; Wang, J.; Liu, Z.; Shang, J.; Wang, X.; Wang, W. GAN-LSTM Joint Network Applied to Seismic Array Noise Signal Recognition. Appl. Sci. 2021, 11, 9987. https://doi.org/ 10.3390/app11219987

Academic Editor: Jun Matsushima

Received: 4 July 2021

Accepted: 12 October 2021

Published: 25 October 2021

Publisher's Note: MDPI stays neutral with regard to jurisdictional claims in published maps and institutional affiliations.

Copyright: (c) 2021 by the authors. Licensee MDPI, Basel, Switzerland. This article is an open access article distributed under the terms and conditions of the Creative Commons Attribution (CC BY) license (https:// creativecommons.org/licenses/by/ $4.0 /)$.
Abstract: The purpose of seismic data processing in nuclear explosion monitoring is to accurately and reliably detect seismic or explosion events from complex ambient noises. Accurate detection and identification of seismic phases are of great significance to the detection and parameter estimation of seismic events. In seismic phase identification, discriminating between noise signals and real seismic signals is essential. Accurate identification of noise signals helps reduce false detections, improves the accuracy of automatic bulletins, and relieves the workload of analysts. At the same time, in seismic exploration, the prime objective in data processing is also to enhance the signal and suppress the noises. In this study, we combined a generative adversarial network (GAN) with a long short-term memory network (LSTM) to discriminate between noise and phases in seismic waveforms recorded by the International Monitoring System (IMS) array MKAR. First, using the beamforming data of the array as the input, we obtained the signal features of seismic phases through the learning of the GAN discriminator network. Then, we input these features and trained the joint network on mixed seismic phase and noise data, and successfully classified seismic phases and noise signals with a recall of $95.28 \%$ and $97.64 \%$, respectively. Based on this model, we established a real-time data processing method, then validated the effectiveness of this method with real 2019 data of MKAR. We also verified whether improved noise signal identification improves the quality of phase association and event detection.

Keywords: GAN; LSTM; seismic array; noise signal recognition

\section{Introduction}

Seismic monitoring, as one of the verifications means identified by the "Comprehensive Test Ban Treaty", is widely applied in the event location, nature discrimination, and yield estimation in nuclear test monitoring due to its sensitivity. The International Data Center (IDC) of the Comprehensive Nuclear-Test-Ban Treaty Organization (CTBTO) collects and processes International Monitoring System (IMS) data in real time and generates a series of seismic events bulletins within the term specified by the treaty [1]. For IMS station data, the IDC first generates a series of standard event lists (SELs) through automatic processing, which is then reviewed and corrected by analysts to generate a reviewed event bulletin (REB).

The purpose of seismic data monitoring in nuclear explosion monitoring is to accurately and reliably detect seismic or explosion events from ambient noises. This processing mainly involves detection, association, location, discrimination, and magnitude/yield estimation [2]. The IMS of CTBTO includes 50 primary seismic stations and 120 auxiliary seismic stations, most of which record seismic signals by the seismic array. The IDC's seismic data processing includes station processing and network association processing [3]. 
The former aims at data space-time filtering, signal detection, feature extraction, and seismic phase identification.

Beamforming and filtering are performed to improve the signal-to-noise ratio (SNR) of array signals. Beamforming [4,5] can improve the signal-to-noise ratio (SNR) and the detection performance by delaying and summing the records of subsites of the seismic array using signal correlation and simultaneously eliminate the effects of non-coherent noise. The short-term average/long-term average (STA/LTA) method capable of reflecting instantaneous array signal energy variations is used to detect seismic signals $[6,7]$. Frequency-wavenumber ( $\mathrm{f}-\mathrm{k}$ ) analysis is applied to estimate the array slowness vector [8]. Polarization analysis is applied to estimate the azimuth and slowness of three-component stations [9]. The Autoregressive Akaike information criterion (AR-AIC) method is applied to accurately estimate the arrival time of seismic phases [10,11]. A method combining rules and a traditional neural network is used to identify seismic phases [3]. Accurate detection and recognition of seismic phases are of great significance to the detection and parameter estimation of final seismic events. In seismic phase recognition, discriminating between noise signals and real seismic signals is an essential step. High false detection rate, as one of the major problems facing the IDC's automatic processing results [12], is mainly attributable to the inaccurate picking and recognition of seismic phases resulting, for example, from the interference of noise signals with real seismic phases. In seismic exploration, although there are different data processing sequences, the prime objective in data processing is still to enhance the signal and suppress the noises [13].

When recording seismic signals, seismic stations also record various noise signals, including instrument noises, man-made noises, wind noises, marine noises, and other noise sources, some being very similar to seismic signals [14]. A list of specific seismic features is extracted from the detected signals. Based on these features, the seismic and noise signals are classified using a rule-based expert system or the traditional BP (backpropagation) neural network algorithm [3]. The rule-based expert system uses signal frequency, horizontal and vertical amplitude ratios, polarization features, and, for arrays, the horizontal slowness, f-k quality, and F-score distributions. It may also use a cascade binary-class classification $\mathrm{BP}$ neural network to classify signals according to the above features. It first distinguishes seismic phases and noise, then further differentiates between teleseismic and regional phases, and finally identifies the regional P or S waves. From 2018 to 2019, 4.5 million noise signals were identified by the IDC automatic processing system, accounting for $54 \%$ of all detected signals. In the interactive analysis, there are many arrivals detected at stations that do not associate to the interactive analysis bulletin LEB. Among these arrivals, most of them are noise signals, partly were corrected as " $\mathrm{N}$ " signals by analysts. This shows that there are still large numbers of noise signals erroneously identified as seismic phase signals in IDC's automatic processing. Accurate removal of all noise signals is an urgent problem to solve to improve the reliability of IDC's automatic bulletins.

In recent years, machine learning, including deep learning, has been widely applied across the fields of computer vision, speech recognition, and natural language processing. The method of deep neural learning was used by many researchers to develop new techniques for seismic phase picking and event detection. For example, Perol used a convolutional neural network (CNN) to detect and locate seismic events in Oklahoma and detected more events than previously cataloged [15]. Ross trained CNNs on picked network data for the Southern California region and proved that this method could estimate P-wave arrival times and first-motion polarities more accurately [16]. Zhu detected P and $S$ phases and noises with U-net and estimated arrival times with probability distribution outputs [17]. Mousavi detected seismic signals by combining CNN with recurrent neural network (RNN) and proved better performance than STA/LTA, template matching, or FAST [18]. Li recognized noise signals and P-wave signals in earthquake early warning systems using GAN and random forest (RF) processes. Their methods can effectively reduce false system alarms [19]. Meier compared the performances of five machine learning methods in recognizing seismic phases from noise signals in earthquake early warning systems 
and showed that their complex deep neural network model based on raw data learning could strongly improve the performance of noise signal recognition [20]. Wu designed a cascaded convolutional neural network capable of detecting different scales of seismic events [21]. Titos designed a deep convolutional network to recognize volcano-seismic events [22]. Zhao applied CNN and U-net to the detection and recognition of Wenchuan earthquake aftershocks and the seismic networks in the capital circle [23,24]. Zhang applied $\mathrm{CNN}$ to locate induced seismicity in Oklahoma [25]. For the global IMS network, Nimar proposed a network association method called NET-VISA based on machine learning to achieve the station's phases association and events detection. NET-VISA mainly uses a probabilistic model and the Bayesian method to find the best set of events explaining the parametric detection observations [26-28].

Noise signal discrimination is a binary classification problem. Deep learning is suitable for solving such classification problems. To execute this, we first need to extract the features from waveform data, then classify the seismic phases and noise signals based on these features. Traditional feature extraction methods require complicated feature engineering. Deep learning provides another solution avoiding feature engineering and has shown good performance on classification problems [29]. At the same time, because beamforming can improve the SNR of seismic array records, we use beamforming data of seismic array as the input to train and test the deep learning network to improve the performance for weak signals further.

In this study, we present a noise discrimination method suitable for array data across the global IMS seismic networks, which combines a generative adversarial network (GAN) with a long short-term memory network (LSTM). First, using beamformed IMS array data as the input, we trained the GAN on a dataset comprised of real seismic $P$ and $S$ phases (Pn, Sn, Pg, Lg, P, PKP) data. After training, the generator of GAN generates these seismic phases data, and at the same time, the discriminator of GAN is considered to have extracted the features of seismic phases. Next, we connected the discriminator with the LSTM to form a new network, and a large volume of 90,000 mixed seismic and noise signals data was used to train the LSTM and fully-connected layer parameters of this network, and eventually, the joint network could realize the classification of seismic phases and noise signals. We show that our new method displays good performance on the test set. The real-time array data processing method established by this model can also identify more noise signals than IDC's method on real 2019 monitoring data of IMS array station MKAR, and it is possible to improve the quality of automatic event detection.

\section{Dataset}

The data used in this study are from the IMS array MKAR. The seismic phase data come from IDC's analyst-reviewed event bulletin (LEB) from 2012 to 2018, including P and S phases such as Pg, Pn, P, PKP, Lg, Sn, and S that have good f-k qualities. Figure 1 shows the distribution of seismic events associated with these phases. Using the arrival time, azimuth, and slowness data corrected by manual analysis, we beamformed the data of subsites at the array. Then we removed the linear trend of the beamformed data, filtered them over the $0.8-3.5 \mathrm{~Hz}$ passband, and used the result as input for the deep neural network. Figure 2 shows the root-mean-square (rms) signal-to-noise ratio (SNR) distribution of the selected dataset, where signal and noise are defined by the rms amplitude in a window $10 \mathrm{~s}$ after and $5 \mathrm{~s}$ before the arrival time, respectively. The noise dataset comprises signals recorded between 2013 and 2015 that are automatically detected and identified as "N" and have not been changed to seismic phases by analysts, as well as those corrected as " $\mathrm{N}$ " by manual analysis. We also limited our selection of noise signals to having no event within $2 \mathrm{~min}$ of the detection time. For noise signals, it is also necessary to beamform the data of subsites of the array. As noise signals do not associate with any event, we can only beamform them according to their automatically detected arrival times, azimuths, and slowness. 


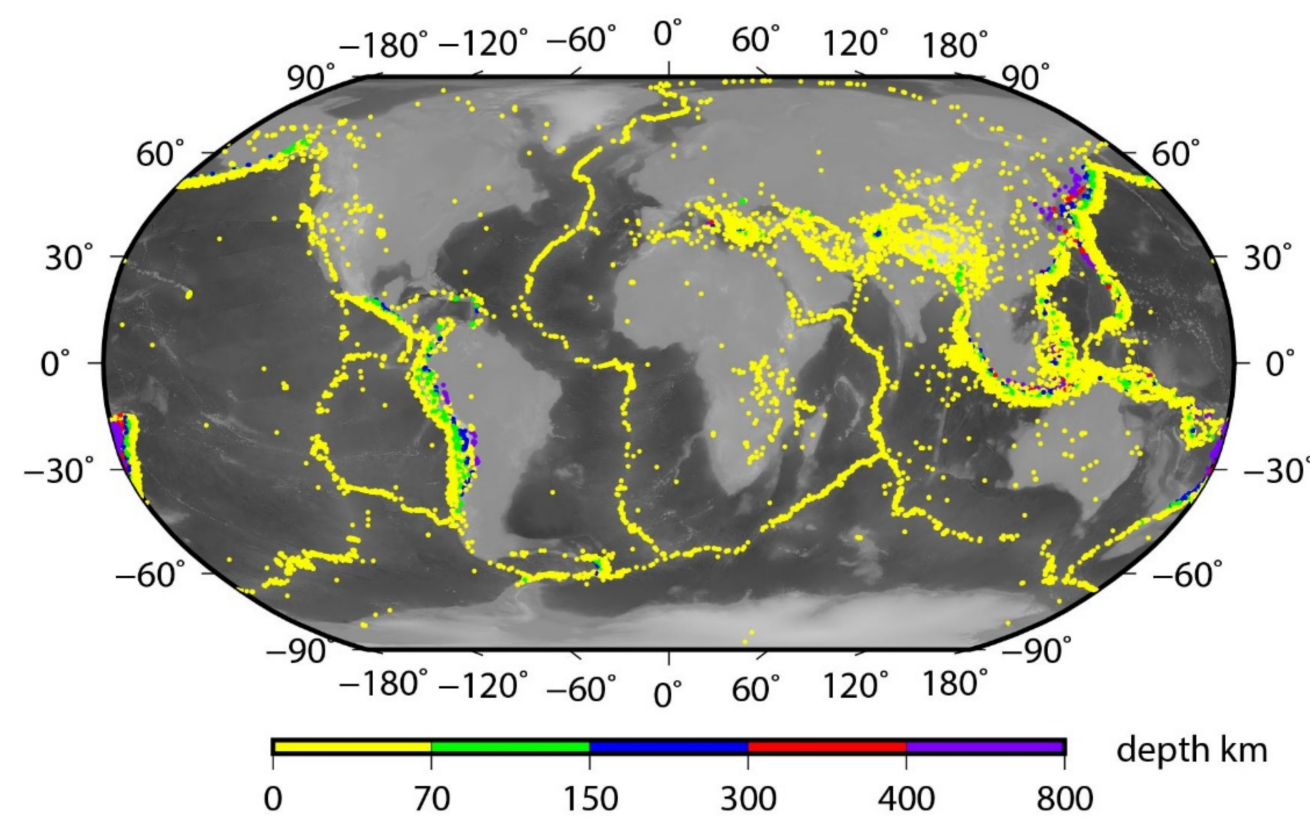

Figure 1. Distribution of IDC LEB events from 2012 to 2018, which associate MKAR phases.

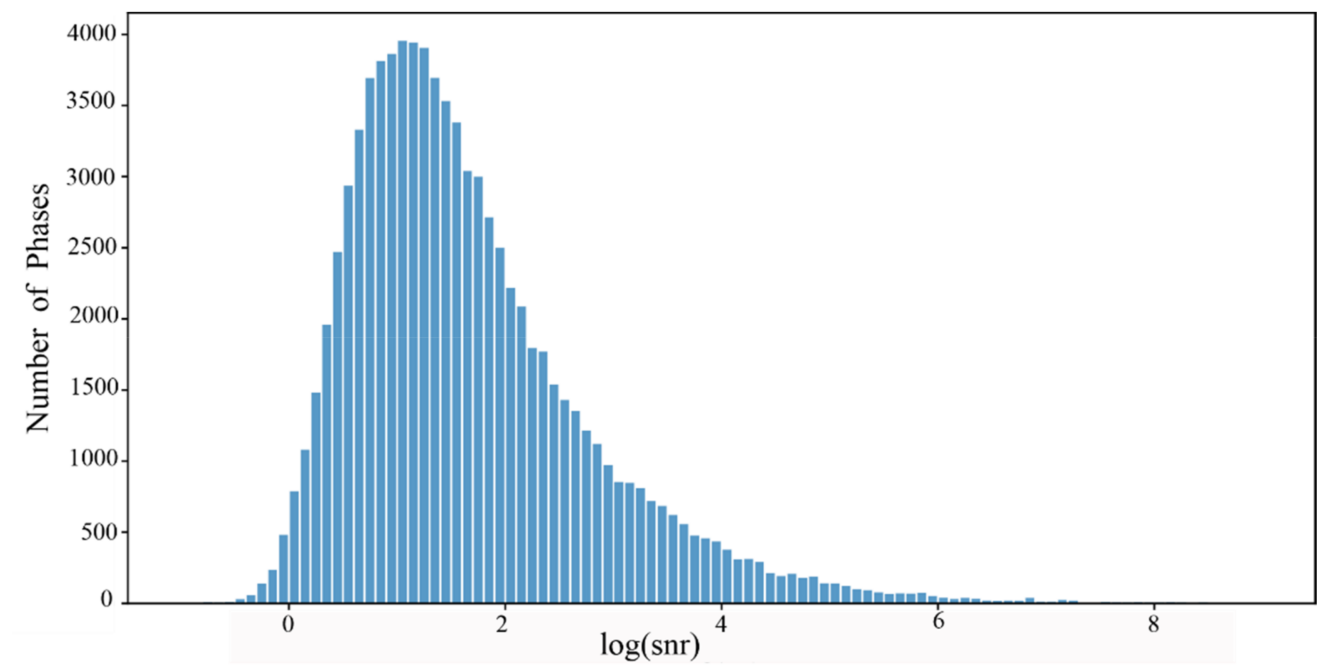

Figure 2. The rms SNR of seismic signals. The window of the signal is 10 s after the arrival time, and the window of noise is $5 \mathrm{~s}$ before the arrival time.

A seismic array is composed of multiple sensors located in a certain range, and the signal-to-noise ratio of far-field signals can be effectively improved by means of delay and sum beamforming. By summing the coherent part of seismic signals at the different array sites using a time delay calculated according to the site's location relative to the reference site and a given slowness vector, the seismic signals are enhanced, and the incoherent noise is canceled out. The theoretical improvement of the SNR of the N element seismic array is be $\sqrt{N}$ through the "delay and sum" process. The beamforming data of MKAR was used to train the joint network to achieve better performance in our method. For historical seismic phase data, the real azimuth and slowness of a phase are obtained based on the final event location and used to calculate the delay of each subsite in the array relative to the reference station, and then the vertical channels data of all subsites are delayed and summed to create the beamforming data of this phase. Figure 3 compares the original waveforms of each site of MKAR with the beamforming data. We train the GAN on the beamforming data of the array, effectively improving the identification ability for weak signals of small-magnitude events. Figure 4 shows the beamformed data of different detected phases. 


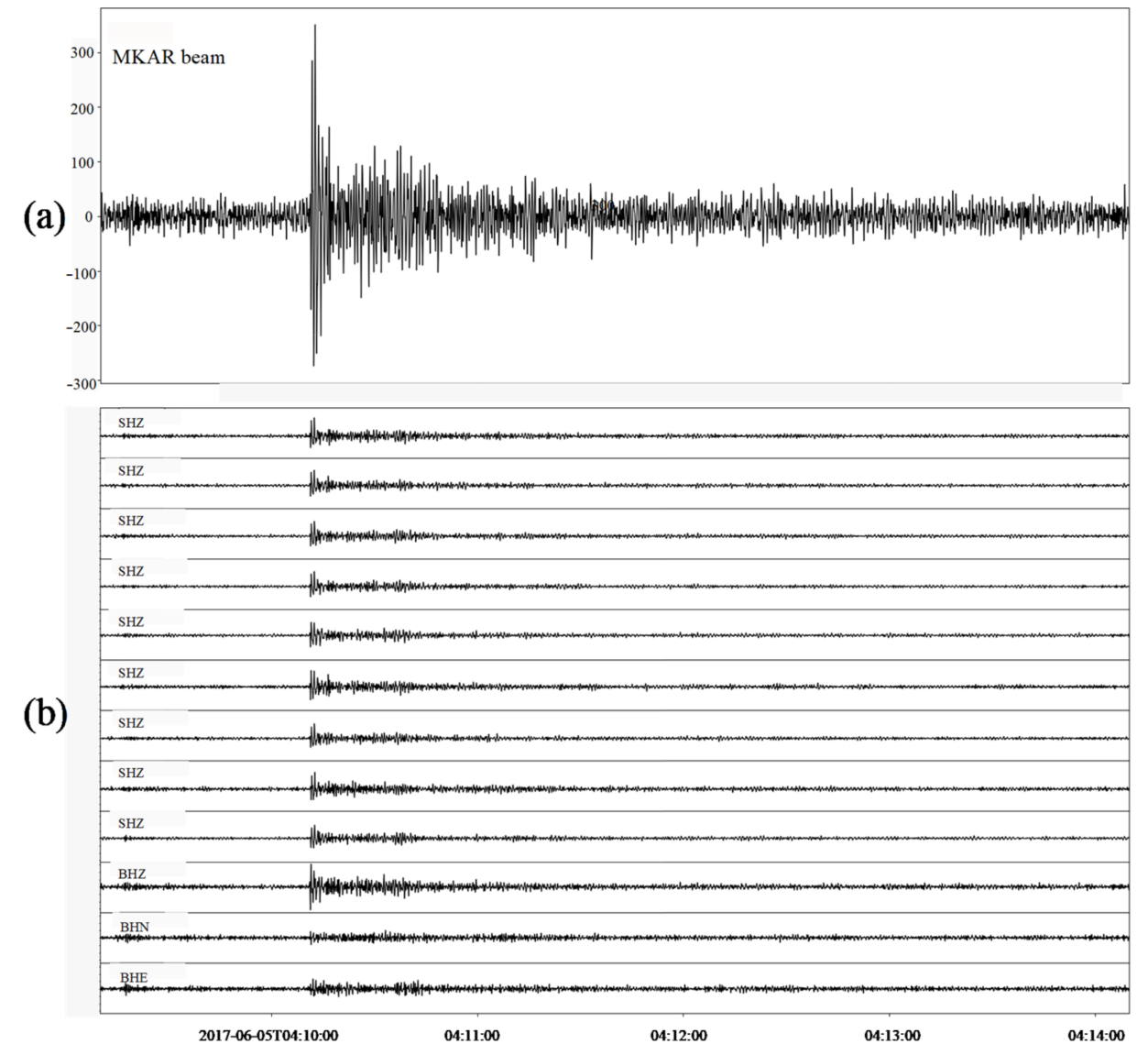

Figure 3. Comparison of the individual subsite waveforms and beamforming waveforms. (a) beamforming waveform. (b) vertical channel waveform of each site of MKAR.
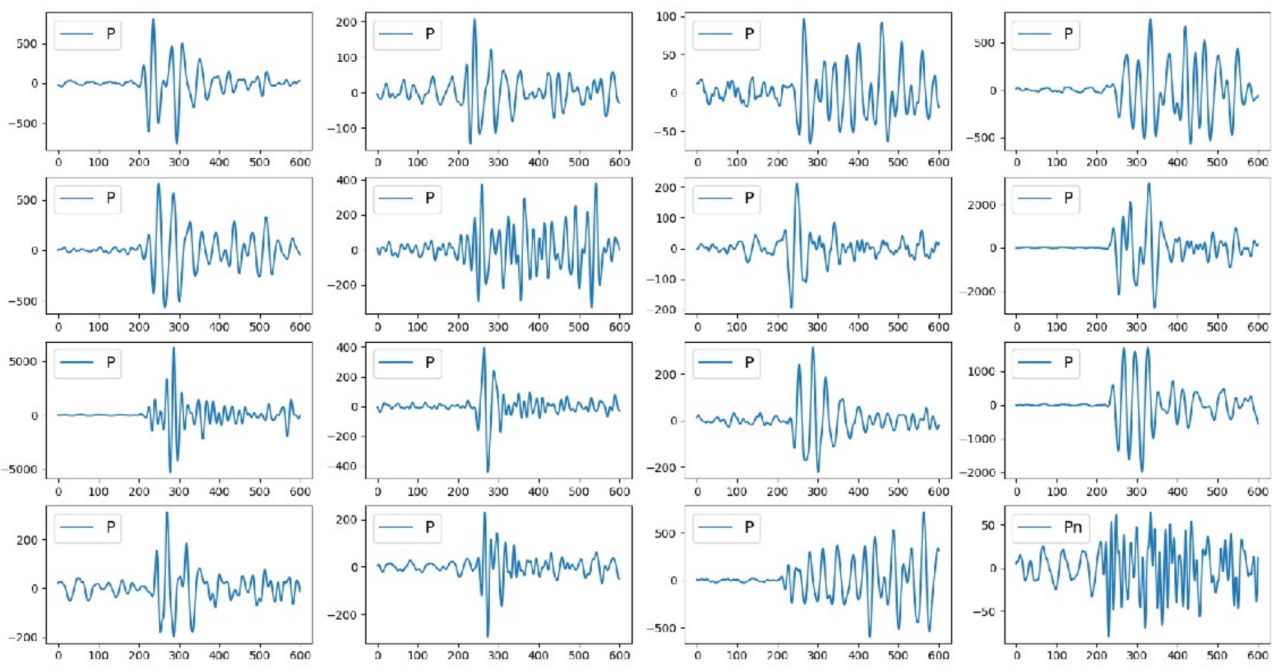

Figure 4. Beamforming data of different phases.

A total of 64,596 seismic phase records were obtained. Table 1 lists the number of each phase. We then preprocessed the data, including (1) truncating the waveform data over a period of $15 \mathrm{~s}$ from $5 \mathrm{~s}$ before to $10 \mathrm{~s}$ after arrival time; (2) linear detrending processing; (3) filtering over the $0.8-3.5 \mathrm{~Hz}$ band; and (4) amplitude normalization. 
Table 1. Types of phases selected and their distributions.

\begin{tabular}{cc}
\hline Phase & Count \\
\hline$P$ & 51,121 \\
\hline Pn & 4083 \\
\hline Lg & 4074 \\
\hline PKP & 2274 \\
\hline Sn & 2001 \\
\hline$P g$ & 784 \\
\hline$S$ & 259 \\
\hline
\end{tabular}

\section{Methodology}

We used a joint GAN-LSTM network to classify and identify seismic phases and noise signals. GAN is an unsupervised learning method developed by Goodfellow [30]. This method is composed of two neural networks, a generator $\mathrm{G}$ and a discriminator D. GAN trains on real seismic signal data. It trains $\mathrm{D}$ to maximize the accuracy of the discriminator model and trains $\mathrm{G}$ to minimize the accuracy of the discriminator model. The two neural networks compete with each other. When the generator has fully learned the distributions of real samples, the network reaches the optimal solution. The GAN discriminator network is a binary classifier of real and false data. After the GAN networks are trained, the convolutional layer part of the discriminator network can serve as a feature extractor of real seismic signals. Figure 5 shows seismic waveforms generated by the GAN generator. We can see that the generator restored the shape of the seismic waveforms quite well. It also indicates the GAN learned the features of the seismic waveforms after training using real seismic data.
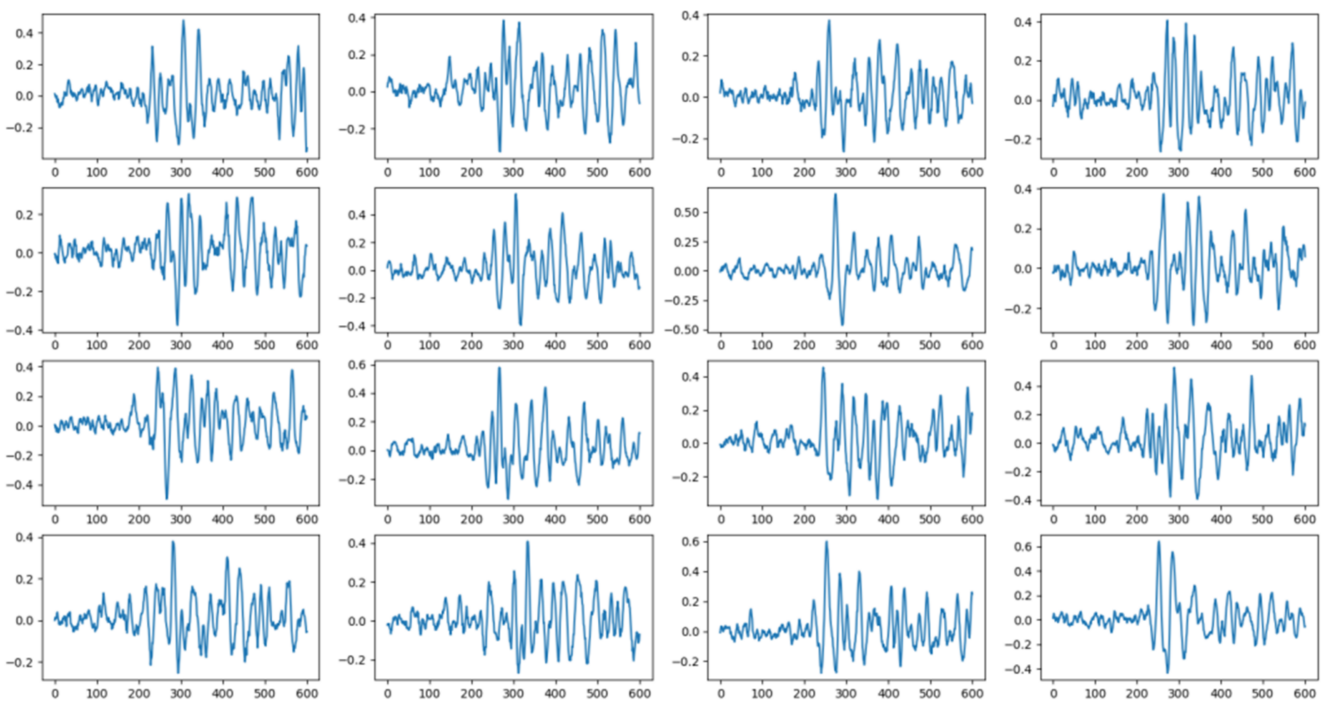

Figure 5. Seismic waveforms generated by the GAN generator.

RNN is a typical deep learning model for processing time sequences. LSTM is one kind of RNN. LSTM network adjusts information flows by introducing the internal mechanism of a "door" and effectively overcomes the short memory of traditional neural networks. RNN is sequence sensitive, but its training speed is slow. Excessively long sequence inputs can lead to gradient disappearance or gradient explosion. With the help of the GAN discriminator, a feature sequence of the original time sequence with limited length is obtained. Using this feature sequence as the input for bidirectional LSTM, the LSTM and fully connected layer parameters are trained on seismic phase data and noise signal data for binary classification. 
In this study, we used the DCGAN [31] architecture as the implementation of GAN. In order to improve the training efficiency of GAN, we adopted the following model construction regime: tanh was used instead of sigmoid as the last layer of the generator; normal distribution was used instead of uniform distribution to sample points in potential spaces; dropouts were used in the discriminator, and random noises were added into their identities; LeakyReLu was used instead of ReLu. We trained the GAN on 15s seismic waveform data (the sampling ratio was 40 , the input data was $600 \times 1$ dimension) as the input. For generator $G$, we used upper sampling plus convolutional layers to replace the pooling layer. On the output layer, we used tanh as the activation function. The input data were 50-dimensional Gaussian noise data. After a series of convolutions, we created $600 \times 1$ one-dimensional waveform data consistent with the size of the training data. For the discriminator D, we use convolutional layers with a step size of 2 to replace the pooling layer; a total of three convolutional layers, and the convolution kernel of each layer was 5. The activation function was LeakyReLU. The slope was 0.2 . The inputs were $600 \times 1$ waveform data. The data dimension was reduced after a series of convolutional layers. The outputs were the binary classification results of seismic signals or noise signals. By connecting the discriminator D to the bidirectional LSTM network, we learned and modeled the sequential pattern of the data. The number of hidden layers on LSTM was set to 128. Finally, we connected the two fully connected layers to classify signals. The sigmoid binary activation function was used for the last fully connected layer. The outputs were the predicted probabilities of each sampling point belonging to seismic or noise. Figure 6 shows the architecture of the network.

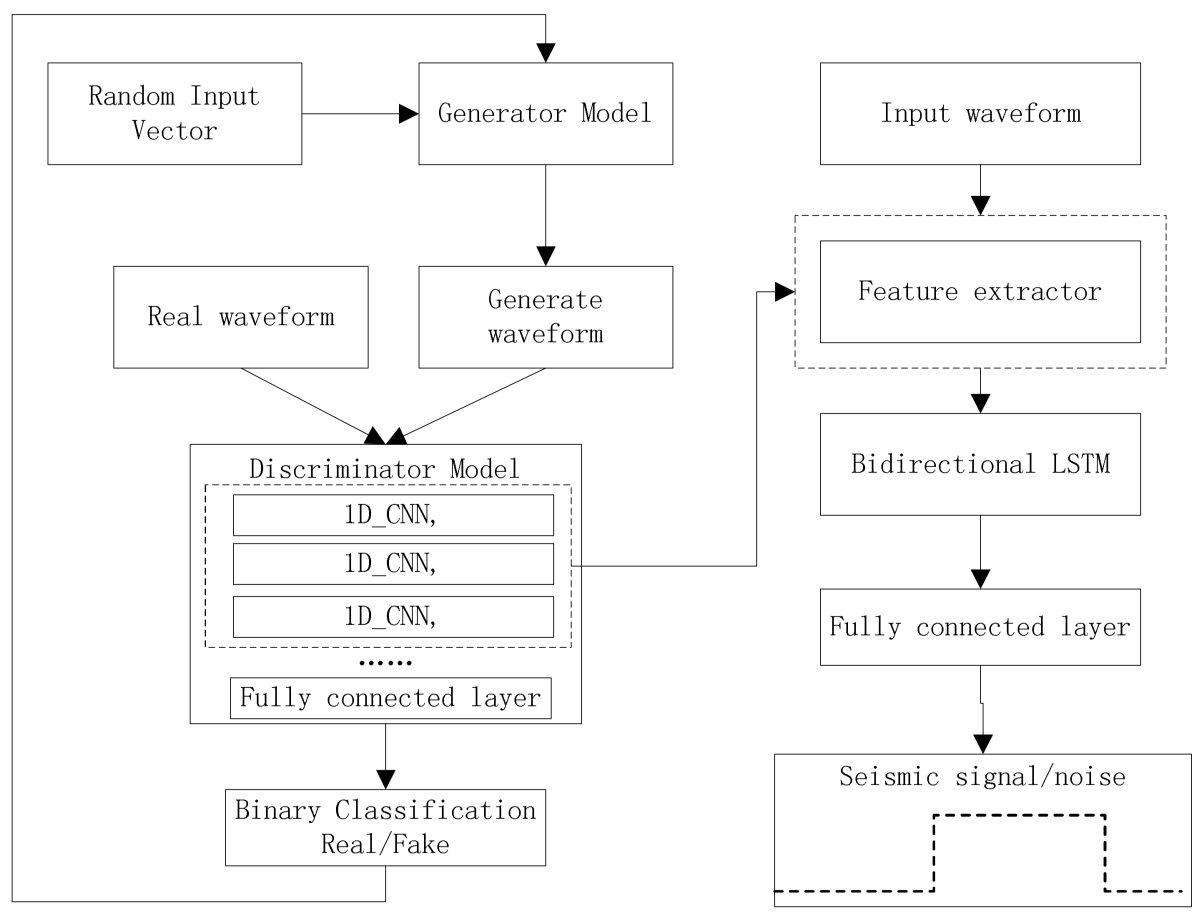

Figure 6. Architecture of the GAN-LSTM joint network (Generative adversarial network, GAN; Long short-term memory network, LSTM). First, real seismic signals are used to train the GAN. After training, the discriminator of GAN is regarded as feature extractors, and the convolutional layers of the discriminator are connected to the LSTM to build the classification network for seismic and noise signals. 


\section{Model Training}

We trained the GAN on 32,000 real seismic phase data, using Adam's stochastic optimization algorithm [32]. The learning ratio was 0.002 . The number of data batches, which controls the data volume used for each iteration, was set to 32. During the training, we first trained the discriminator on generated seismic signals and real seismic signals, then trained the generator with random inputs and the trained discriminator; thus, we froze the discriminator and trained the generator to cheat the discriminator.

We used an NVIDIA TITANV graphic processing unit for processing the graphs. The training was terminated after 9054 iterations. The entire process took $20 \mathrm{~min}$. Figure 7 shows the variations in generator network loss and discriminator network loss with a number of training rounds.

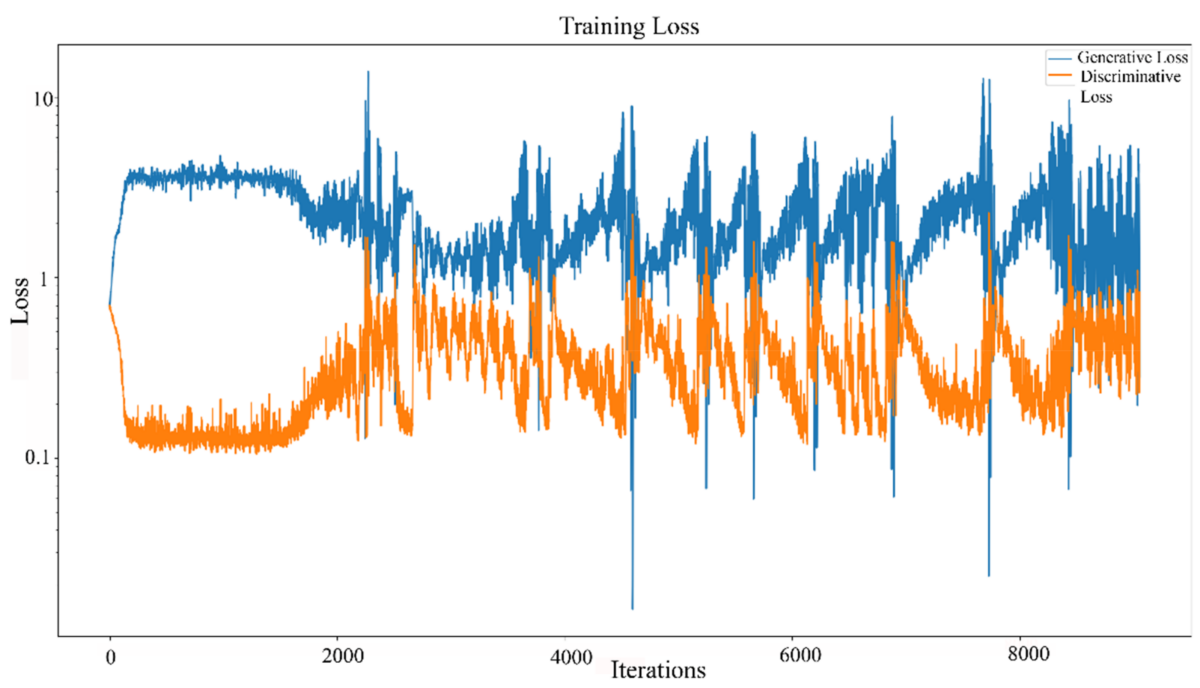

Figure 7. GAN network training loss profile.

Figure 5 in Section 3 shows the seismic waveform data distributions generated by the trained generator. After training, the generator can produce a signal that is very similar to the real seismic signal. It can be seen the amplitude obviously increases at the phase arrival time (5s). This shows that the GAN training is finished and also suggests that the GAN learned the generic features of the seismic phase waveforms, which can be further used in subsequent classification tasks.

We connected the trained GAN discriminator to the bidirectional LSTM network into a new joint noise recognition network, then trained it on 90,000 mixed seismic signals and noise signals. The data set included 45,000 seismic phases and 45,000 noise signals, with $80 \%$ belong to the training set and $20 \%$ to the validation set. The training was performed by fixing the GAN discriminator network parameters and only training the parameters of the LSTM and fully connected network. Again, an NVIDIA TITANV graphic processing unit was used for processing graphs. The learning was terminated when the loss of the validation data set was no longer reduced in the previous five rounds of training. The parameter with the minimum loss on the validation data set was used as the final parameter of the model.

Figure 8 shows the reduction in the loss with a number of epochs. We can see that the loss of the validation data set after the 16th epoch dropped to 0.0844 . At that point, the recognition accuracy of the validation set was $96.95 \%$. The training took about $10 \mathrm{~min}$. 


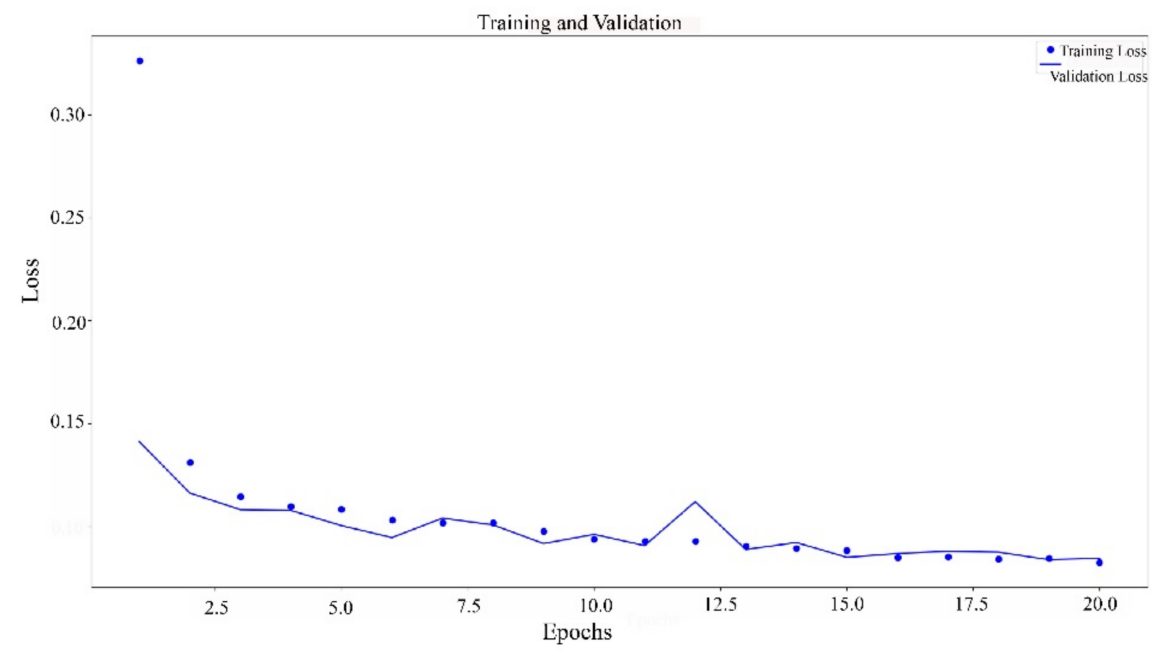

Figure 8. Variation in loss function with number of iterations.

\section{Results}

We tested the model with another 20,000 test data, including approximately 10,000 real phase signals and 10,000 noise signals. Figure 9 shows the recognition results of some of the seismic/noise signals. The outputs are the class probabilities at each single sampling point: 1 denotes a seismic phase, and 0 denotes a noise signal. We can see that all classification results are correct, demonstrating the ability to identify seismic phases and noise signals accurately and to give the arrival times of seismic phases accurately.
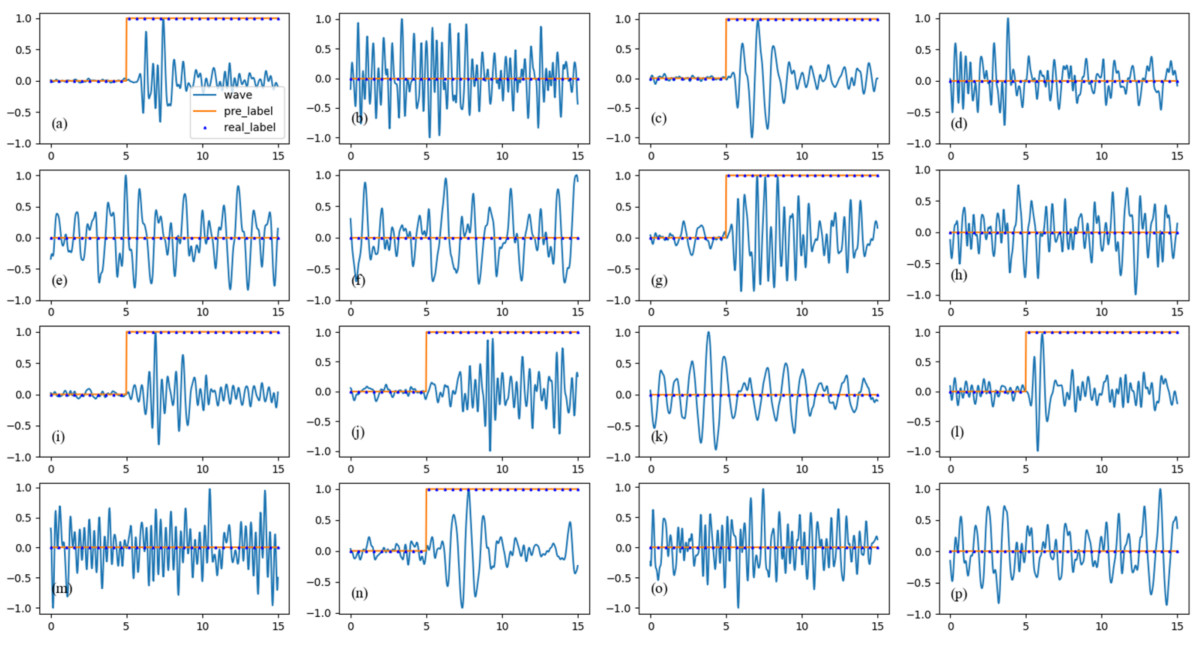

Figure 9. Signal prediction results of the test data set versus real results (In the $\mathbf{a}-\mathbf{p}$ subfigure, the blue "..." denotes a real signal category,1 means seismic phase, 0 means noise signal; the yellow "-" denotes a model-predicted signal category. All the predicted results are consistent with actual results.).

We defined the confusion matrix of the classification of seismic signals and noise signals as shown in Table 2.

Table 2. Classification confusion matrix.

\begin{tabular}{cccc}
\hline Predicted & Real & Signal & Noise \\
\hline Signal & & TP & FN \\
\hline Noise & FP & TN \\
\hline
\end{tabular}


We then used precision, recall, and F-score to further evaluate the performance of the model according to the definition formulas below:

$$
\begin{gathered}
\text { Precision }=\frac{T P}{T P+F P} \text { Recall }=\frac{T P}{T P+F N} \\
F=\frac{2 \times \text { Precision } \times \text { Recall }}{\text { Precision }+ \text { Recall }}
\end{gathered}
$$

Figure 10 shows the P-R curves resulting from precision and recall calculation with different thresholds. As the threshold varies within the $[0,1]$ interval, the recall spans from $76 \%$ to $100 \%$; the precision spans from $48 \%$ to $100 \%$. When the threshold is in the $[0.3,0.7]$ interval, the recall is greater than $95 \%$; the precision is greater than $93 \%$. Figure 11 shows the variation in the F-score with the threshold. When the threshold is set to 0.67 , the maximum F-score is 0.9636 . At that point, the recall and precision are $97.46 \%$ and $95.28 \%$ for seismic signals and $97.64 \%$ and $95.60 \%$ for noise signals, which indicate that the algorithm reached a good classification performance for seismic phases and noise signals.

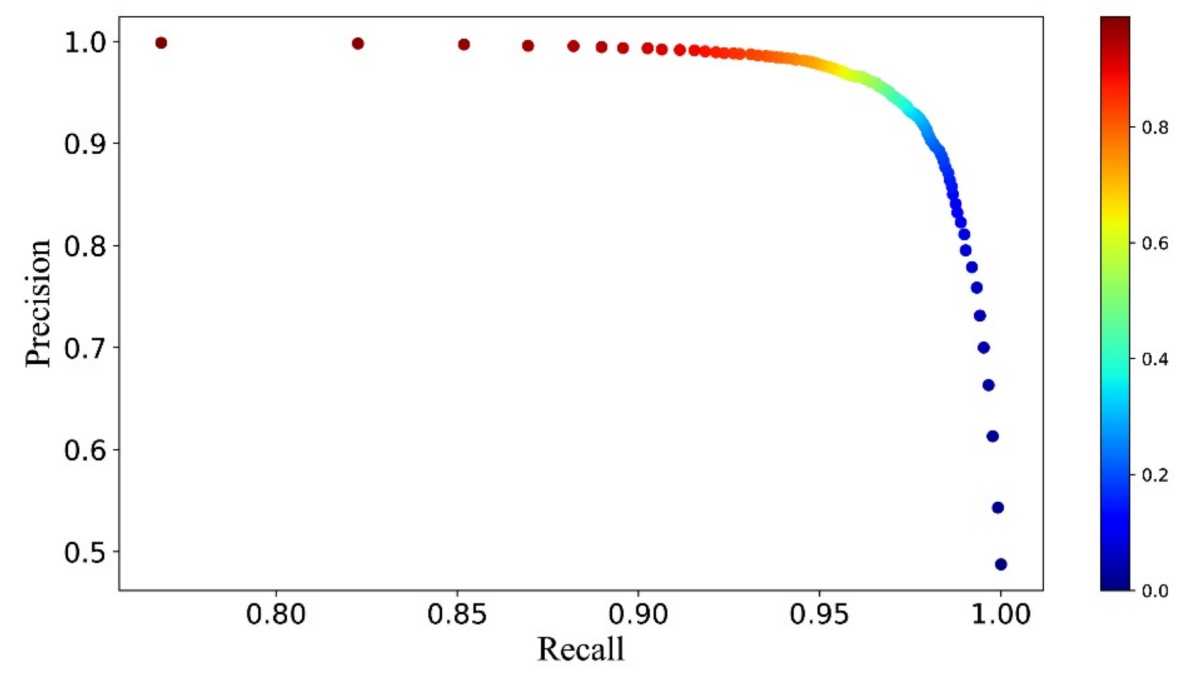

Figure 10. Precision-recall curve.

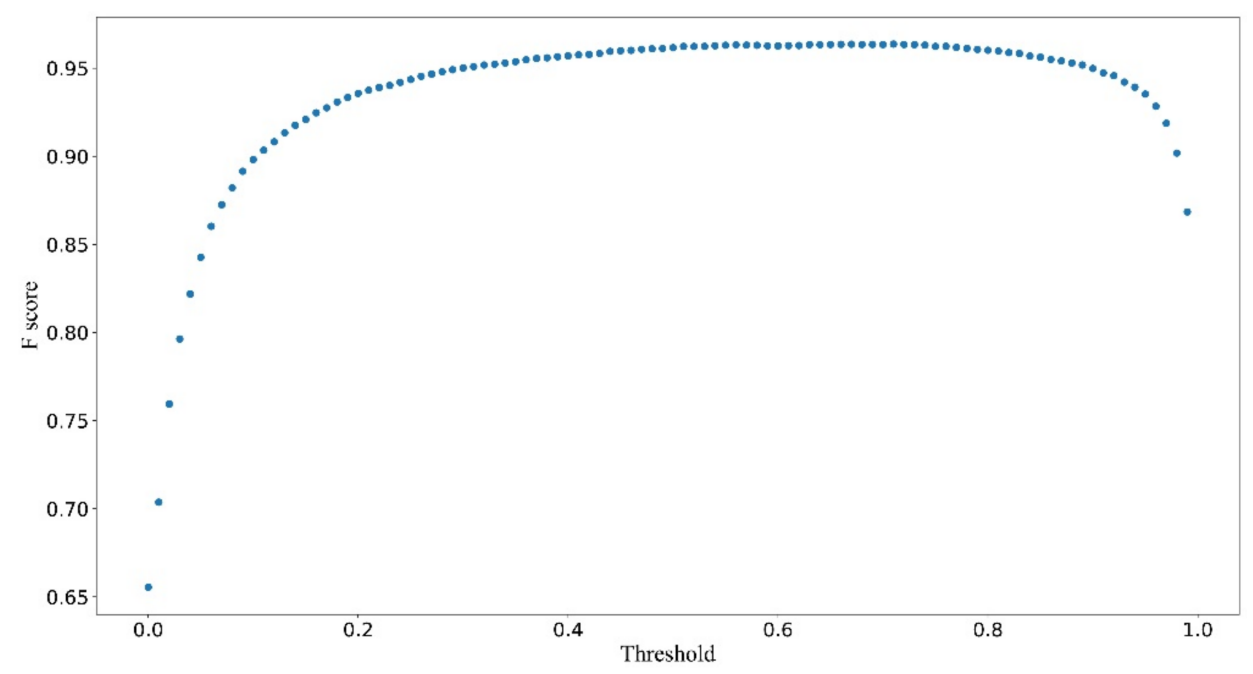

Figure 11. Threshold-F curve. 


\section{Application to New Monitoring Data}

We tested the effectiveness of this new method with new monitoring data by establishing a real-time array data noise processing procedure. First, we retrieved the original waveform data of the MKAR array beamform and filtered them with the previously described beamforming parameters, and detected signals with STA/LTA on several beamformed passbands. Next, we selected the maximum SNR as the final detection, obtained the optimal azimuth and slowness intervals, and calculated the accurate azimuth and slowness by f-k analysis. For each detection, we truncated the data $5 \mathrm{~s}$ before and $10 \mathrm{~s}$ after the arrival time, re-beamformed them with the azimuth and slowness information estimated by $\mathrm{f}-\mathrm{k}$ analysis, filtered them over the $0.8-3.5 \mathrm{~Hz}$ passband, and normalized them. We input the normalized beamformed data into the trained network model and tested them, with a probability threshold of 0.67 , to determine whether the detected signals were seismic phases or noises. Then we applied these phases in event association to form events. Figure 12 shows the real-time monitoring data processing procedure.

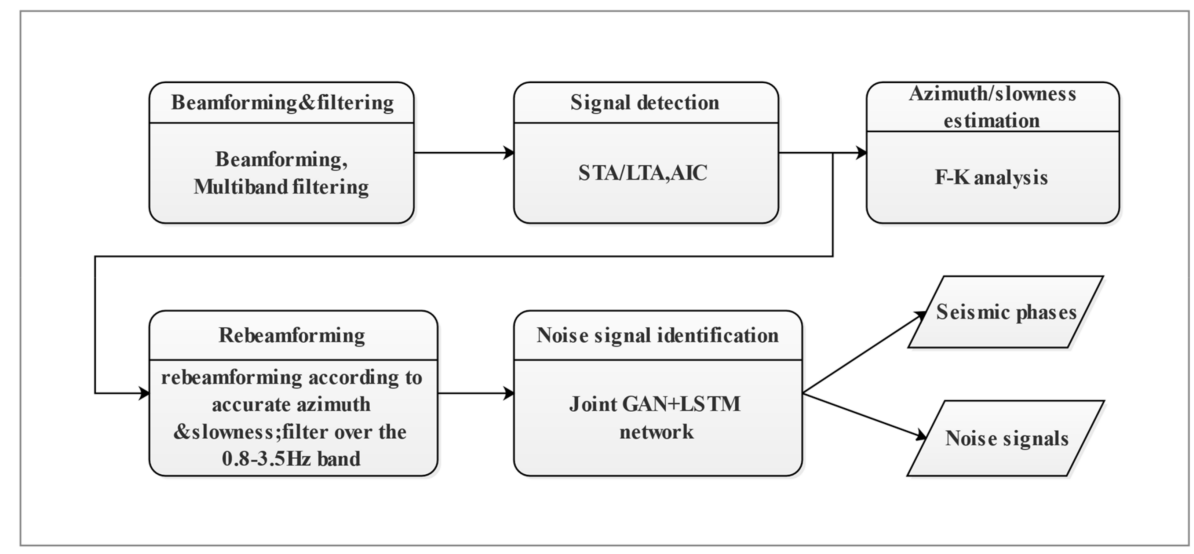

Figure 12. Real-time monitoring array data processing procedure.

We tested the model with the half-year phase data of 2019, involving 5376 seismic phases and 6439 noise signals. Besides seismic phases used in training above, 27 types of phases, including PKP and PKPbc, were used. Figure 13 shows the top ten phase types by the number of phases. We processed the half-year data with the above method, and the result was an identification rate of $90.8 \%$ for seismic phases and $97.8 \%$ for noise signals. Figure 14 shows the identification results of some of the seismic phases.

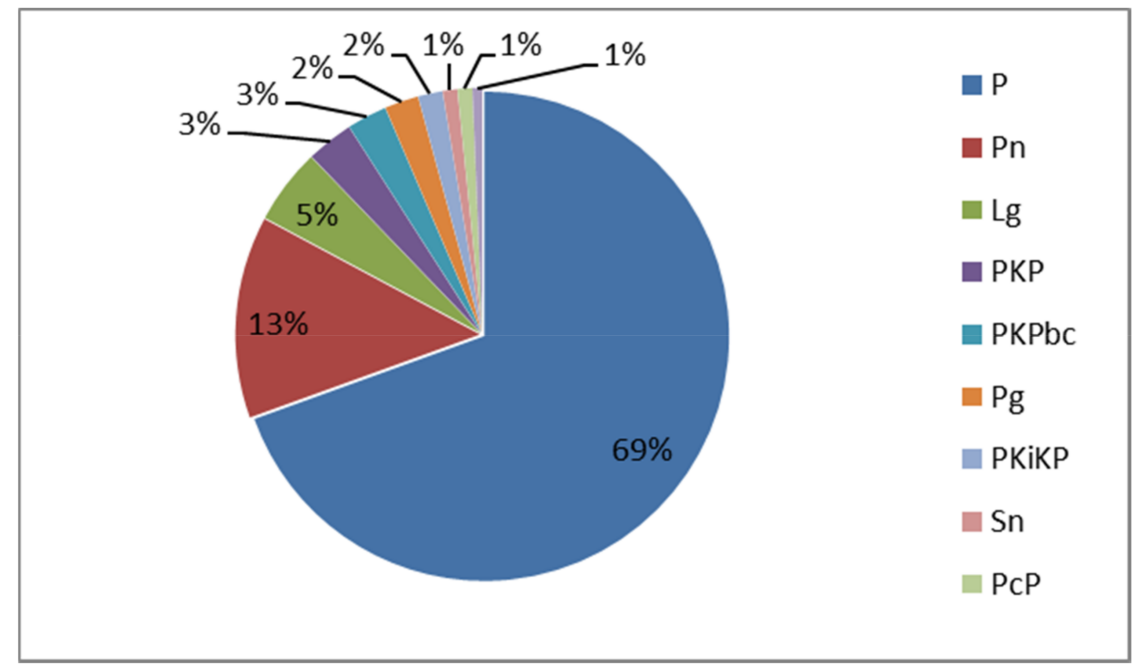

Figure 13. The distribution of top 10 phases of MKAR in 2019 half year events. 

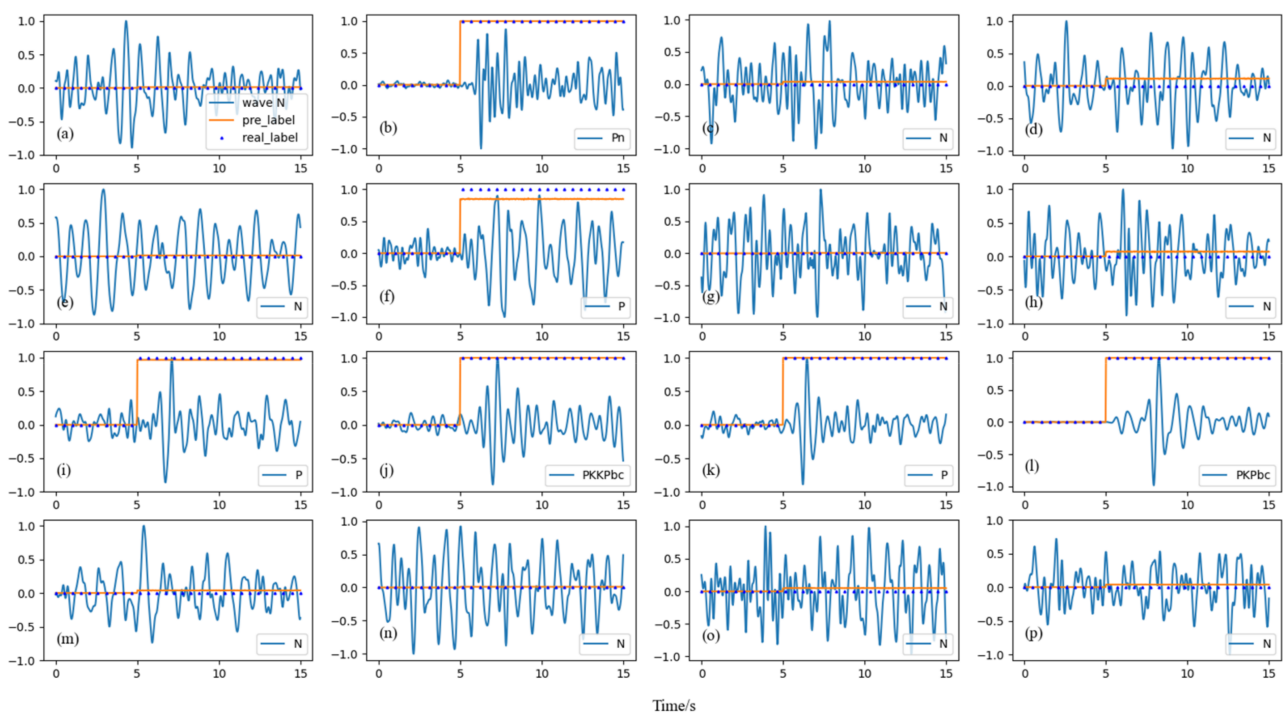

Figure 14. The identification results of MKAR seismic phases. (In the a-p subfigure, the blue " ... " denotes a real signal category,1 means seismic phase, 0 means noise signal; the yellow "_" denotes a model-predicted signal category. The predicted results of these samples are consistent with actual results.).

In order to further test the performance of our method for the automatic detection signals, we compared our method with the traditional identification method in IDC on the part of the MKAR automatic detection dataset in 2019. There are 17,060 samples of automatic signal detection, and the identified results of the IDC automatic identification algorithm are 10,676 seismic phases and 6930 noise signals. There are 7674 seismic phases and 9836 noise signals identified by our method, 2456 more noise signals than IDC. Most of these 2456 noise signals are identified as seismic phases in the IDC method, and some of these noise signals are used to form automatic events in IDC. However, compared with the seismic phases associated with interactive analysis events (LEB), 9023 out of 9386 noise signals identified by our method are not used to form real seismic events, account for $96.13 \%$ of the total noise signals. It shows that our method is able to identify more noise signals, which are misidentified as seismic phases and used to form false events in IDC. It also shows that the use of this method for automatic seismic phase identification in automatic processing reduces the false events in the automatic bulletin.

\section{Analysis and Discussion}

We trained the GAN on beamformed data of an array by taking advantage of its unsupervised learning ability, enabling the GAN to learn seismic data features. We connected the discriminator network to the LSTM network into a classifier, trained this network on seismic and noise data, fixed the parameters of the discriminator network and only trained the LSTM network parameters, and finally, successfully achieved the seismic signals and noise signals classification. First, the GAN discriminator network was composed of several convolutional layers, enabling it to extract the features of seismic signals effectively. After rounds of training, we compared the seismic signal waveforms generated by the GAN generator network with the original waveforms to determine whether the GAN discriminator network learned the features of these data. LSTM can effectively process sequence data. If original waveform data are directly inputted, as the data volume is large, the training speed will be too slow. When the data features extracted by the GAN discriminator network are inputted into the LSTM, not only the input data volume is reduced, the major features of the data are also retained. At the same time, for the GAN, because the discriminator is the result of competing with the generator, the performance would be better than the simple $\mathrm{CNN}$ in terms of feature extraction. 
Traditional detection methods usually divide the monitored band range into a number of narrow bands to perform detection and identification in each of these narrow bands, then combine the results of multiple bands. After statistical analysis of the monitoring 10-year signals at MKAR, we can see that in the above detection band, the SNR of noise signals is sometimes greater than the detection threshold, but in a broad band of $0.8-3.5 \mathrm{~Hz}$, the signal energy is commonly low. As the convolutional layers of deep CNN are intended to learn local patterns, they can examine each small piece of the neural network more intensively to obtain more abstract features, and the entire CNN can learn the spatial hierarchies of features. For this reason, we used the GAN discriminator network to perform the feature extraction function, allowing intensive analysis of different dimensional features of the input waveform data. Although it does not perform narrow-band filtering of the input data as the traditional detection methods perform, it can still obtain satisfactory classification and identification results.

In this study, first, we trained the GAN on phase features, using its discriminator network as a feature extractor and its generator network as a generator of new phase data. The IDC automatically detected phases include regional $\mathrm{P}$ and $\mathrm{S}$ phases and teleseismic phases. From the phases listed in Table 1, we can see that the number of phases distributions is very uneven. In order to apply the deep learning method to IDC phase identification, a large volume of training data with roughly equal identified data distribution is required. In this sense, the GAN generator network can play a role. For example, if we train the GAN on data of a type of phase, we can generate data of this type of phase. These data possess the typical features of this type of phase but are not completely the same and have a degree of randomness. It is similar to the rotation, cropping, and change in color in image processing techniques, which can be used as a data enhancement method. The generated phase data can effectively enlarge the training data set and improve the performance of the model.

In the records of IMS stations, there are teleseismic, regional, and local phases, among which the number of teleseismic phases is far more than that of local phases. In our study, local phases (Pg, Lg) accounted for 7.5\%. Thus, the GAN-LSTM joint network trained in our work should be effective in the local phases. If you wish to pursue the best results in seismic exploration or other areas, you can use the records from these areas to fine-tune or retrain the network. After retraining, the network would show good recognition performance for signals of these fields.

\section{Conclusions}

We established a joint GAN + LSTM network for identifying seismic array noise signals. We used the GAN discriminator network to learn the features of seismic phases, then input these features into the LSTM network and, by training the joint network, successfully identified and classified seismic phases and noise signals. The following understandings were obtained:

(1) Using beamforming data of regional seismic and teleseismic $\mathrm{P}$ and $\mathrm{S}$ phases of an array, the GAN can effectively learn the main features of seismic phases. Its discriminator can be used to extract features of seismic phases;

(2) By training the GAN discriminator and LSTM joint network on mixed seismic and noise signal data while fixing the discriminator network parameters and training only the LSTM network parameters, we can achieve the reliable classification of seismic phases and noise signals, with $97.46 \%$ recall and $95.28 \%$ precision for seismic signal identification and classification;

(3) The real-time monitoring array data processing method developed by this model can classify IDC seismic phases and noise signals. The test with half-year data of 2019 shows that the identification rate of seismic phases is $94.12 \%$, and $95.03 \%$ for noise signals. Improved noise signal identification performance can further improve the quality of phase association and event detection. 
Author Contributions: Conceptualization, D.H. and X.W.; methodology, J.L. and G.C.; simulation and testing, M.H. and J.W.; data collection, Z.L. and J.S.; writing-review and editing, W.W. All authors have read and agreed to the published version of the manuscript.

Funding: This research received no external funding.

Informed Consent Statement: Informed consent was obtained from all subjects involved in the study.

Acknowledgments: It is grateful to all colleagues who have contributed to this research.

Conflicts of Interest: The authors declare no conflict of interest.

\section{References}

1. Coyne, J.; Bobrov, D.; Bormann, P.; Duran, E.; Grenard, P.; Haralabus, G.; Kitov, I.; Starovoit, Y. CTBTO: Goals, networks, data analysis and data availability. In New Manual of Seismological Observatory Practice 2 (NMSOP-2); Bormann, P., Ed.; Deutsches Geo ForschungsZentrum GFZ: Potsdam, Germany, 2012; pp. 1-41.

2. Maceira, M.; Blom, P.S.; MacCarthy, J.K.; Marcillo, O.E.; Euler, G.G.; Begnaud, M.L.; Slinkard, M.E. Trends in Nuclear Explosion Monitoring Research \& Development-A Physics Perspective. In Trends in Nuclear Explosion Monitoring Research E Development—A Physics Perspective (No. LA-UR-17-21274); Los Alamos National Lab. (LANL): Los Alamos, NM, USA, 2017; p. 2017. [CrossRef]

3. Le Bras, R.; Wuster, J. IDC processing of seismic, hydroacoustic and infrasonic data. Revision 1. In IDC Documentation User Guides; CTBTO: Vienna, Austria, 2002.

4. Rost, S.; Thomas, C. Array seismology: Methods and applications. Rev. Geophys. 2002, 40, 1003. [CrossRef]

5. Schweitzer, J.; Fyen, J.; Mykkeltveit, S.; Gibbons, S.J.; Pirli, M.; Kühn, D.; Kværna, T. Seismic arrays. In New Manual of Seismological Observatory Practice-2(NMSOP-2); Geo ForschungsZentrum GFZ: Potsdam, Germany, 2012; Chapter 9. [CrossRef]

6. Allen, R. Automatic earthquake recognition and timing from single traces. Bull. Seism. Soc. Am. 1978, 68, 1521-1532. [CrossRef]

7. Allen, R. Automatic phase pickers: Their present use and prospects. Bull. Seism. Soc. Am. 1982, 72, S225-S242. [CrossRef]

8. Kværna, T.; Doornbos, D.J. An integrated approach to slowness analysis with arrays and three-component stations. NORSAR Semiannu. Technucal. Summ. 1985, 1, 2-85.

9. Jurkevics, A. Polarization analysis of three-component array data. Bull. Seism. Soc. Am. 1988, 78, $1725-1743$.

10. Akaike, H. A new look at the statistical model identification. IEEE Trans. Autom. Control 1974, 19, 716-723. [CrossRef]

11. Maeda, N. A method for reading and checking phase times in auto processing system of seismic wave data. Zisin 1985, 38, 365-379. [CrossRef]

12. Draelos, T.J.; Ballard, S.; Young, C.J.; Brogan, R.A. Refinement and testing of the probabilistic event detection, association, and location algorithm. In Proceedings of the 2012 Monitoring Research Review: Ground-Based Nuclear Explosion Monitoring Technologies, Albuquerque, NM, USA, 10 September 2012; National Nuclear Security Administration, I(LA-UR-12-24325). pp. 221-231.

13. Mondol, N.H. Seismic Exploration. In Petroleum Geoscience; Springer: Berlin/Heidelberg, Germany, 2010. [CrossRef]

14. Fu, S.F.; Liu, B.C. Seismology Course; Seismological Press: Beijing, China, 1991.

15. Perol, T.; Gharbi, M.; Denolle, M. Convolutional neural network for earthquake detection and location. Sci. Adv. 2018, 4, e1700578. [CrossRef]

16. Ross, Z.E.; Meier, M.-A.; Hauksson, E. P-wave arrival picking and first-motion polarity determination with deep learning. J. Geophys. Res. Solid Earth 2018, 123, 5120-5129. [CrossRef]

17. Zhu, W.; Beroza, G.C. PhaseNet: A deep-neural-network-based seismic arrival-time picking method. Geophys. J. Int. 2018, 216, 261-273. [CrossRef]

18. Mousavi, S.M.; Zhu, W.; Sheng, Y.; Beroza, G.C. CRED: A Deep Residual Network of Convolutional and Recurrent Units for Earthquake Signal Detection. Sci. Rep. 2019, 9, 10267. [CrossRef]

19. Li, Z.; Meier, M.A.; Hauksson, E.; Zhan, Z.; Andrews, J. Machine Learning Seismic Wave Discrimination: Application to Earthquake Early Warning. Geophys. Res. Lett. 2018, 45, 4773-4779. [CrossRef]

20. Meier, M.A.; Ross, Z.E.; Ramachandran, A.; Balakrishna, A.; Nair, S.; Kundzicz, P.; Yue, Y. Reliable Real-Time Seismic Signal/Noise Discrimination with Machine Learning. J. Geophys. Res. Solid Earth 2018, 124. [CrossRef]

21. Wu, Y.; Lin, Y.; Zhou, Z.; Bolton, D.C.; Liu, J.; Johnson, P. DeepDetect: A cascaded region-based densely connected network for seismic event detection. IEEE Trans. Geosci. Remote Sens. 2018, 57, 62-75. [CrossRef]

22. Titos, M.; Bueno, A.; Garcia, L.; Benitez, C. A deep neural networks approach to automatic recognition systems for volcano-seismic events. IEEE J. Sel. Top. Appl. Earth Obs. Remote Sens. 2018, 11, 1533-1544. [CrossRef]

23. Ming, Z.; Shi, C.; Yuen, D. Waveform classification and seismic recognition by convolution neural network. Chin. J. Geophys. Chin. Ed. 2019, 62, 374-382. [CrossRef]

24. Ming, Z.; Shi, C.; LiHua, F. Earthquake phase arrival auto-picking based on U-shaped convolutional neural network. Chin. J. Geophys.-Chin. Ed. 2019, 62, 3034-3042. [CrossRef]

25. Zhang, X.; Zhang, J.; Yuan, C.; Liu, S.; Chen, Z.; Li, W. Locating induced earth quakes with a network of seismic stations in Oklahoma via a deep learning method. Sci. Rep. 2020, 10, 1-12. 
26. Nimar, S.; Arora, S.R.; Erik, S. NET-VISA: Network Processing Vertically Integrated Seismic Analysis. Bull Seismol. Soc. Am. 2013, 103, 709-729. [CrossRef]

27. Le Bras, R.; Arora, N.; Kushida, N.; Mialle, P.; Bondár, I.; Tomuta, E.; Taylor, T. NET-VISA from cradle to adulthood. A machine-learning tool for seismo-acoustic automatic association. Pure Appl. Geophys. 2020. [CrossRef]

28. Li, J.; Wang, J.; Wang, X.; Jiang, C.; Wang, W.; Liu, J. Evaluation and comparison of the results of the NET-VISA seismic event association method based on Bayesian theory. Nat. Hazards 2021, 105, 1521-1539. [CrossRef]

29. LeCun, Y.; Bengio, Y.; Hinton, G. Deep learning. Nature 2015, 521, 436-444. [CrossRef]

30. Goodfellow, I.; Pouget-Abadie, J.; Mirza, M.; Xu, B.; Warde-Farley, D.; Ozair, S.; Bengio, Y. Generative adversarial nets. In Proceedings of the 27th International Conference on Neural Information Processing Systems_Volume 2 (NIPS'14); MIT Press: Cambridge, MA, USA, 2014; pp. 2672-2680.

31. Radford, A.; Metz, L.; Chintala, S. Unsupervised Representation Learning with Deep Convolutional Generative Adversarial Networks. arXiv 2015, arXiv:1511.06434.

32. Kingma, D.P.; Ba, J. Adam: A Method for Stochastic Optimization. In Proceedings of the 3rd International Conference on Learning Representations (ICLR 2015), San Diego, CA, USA, 7-9 May 2015. 